\title{
Separation and Quantification of 1,4-benzodiazepines: HPLC versus CZE
}

\author{
Květa Kalíková, ${ }^{a, *}$ Martina Riesová, ${ }^{a}$ Richard Chudoba, ${ }^{a}$ Martin G. Schmid, ${ }^{b}$ and Eva Tesařová ${ }^{a}$ \\ ${ }^{a}$ Department of Physical and Macromolecular Chemistry, Faculty of Science, Charles University in Prague, \\ 12843 Prague 2, Albertov 2030, Czech Republic \\ ${ }^{\mathrm{b}}$ Department of Pharmaceutical Chemistry, Institute of Pharmaceutical Sciences, Karl-Franzens-University Graz, \\ Universitätsplatz 1, A-8010 Graz, Austria
}

\begin{abstract}
The goal of the present study was to separate a set of benzodiazepines, namely bromazepam, oxazepam, nitrazepam, chlordiazepoxide, flunitrazepam, lormetazepam and diazepam by analytical scale HPLC and CZE. The both methods for separation of these seven compounds from the 1,4-benzodiazepine group were optimized and compared. LODs and LOQs were determined under the optimized conditions in the both methods. The corresponding LOD and LOQ values are approximately three orders of magnitude lower in HPLC than in CZE. As expected, elution order was found to be different for the both techniques. As a result of a critical collation of all the parameters considered, RP-HPLC was found to be more suitable for determination of the set of benzodiazepines. A real sample analysis was performed under optimized conditions to demonstrate applicability of the proposed analytical methods. (doi: $10.5562 / \mathrm{cca} 1738$ )
\end{abstract}

Keywords: RP-HPLC, CZE, benzodiazepines, pharmaceuticals

\section{INTRODUCTION}

1,4-benzodiazepines (BZDs) are a well-known class of psychoactive drugs; they are known primarily for their hypnotic and sedative effects. ${ }^{1}$ This pharmaceutical group is also used as anxiolytics, anticonvulsants and muscle relaxants. ${ }^{2}$ They are still widely prescribed but also abused. ${ }^{3,4}$ Many studies on these drugs can be found in the literature. ${ }^{5-8}$ Diverse analytical methods for determination of benzodiazepines in various matrices have been published, ${ }^{9,10}$ e.g. potentiometry, ${ }^{11}$ immunoassays, ${ }^{12}$ spectrophotometry ${ }^{13}$ and also separation techniques that represent a clear majority of the reported methods because they allow determination of individual compounds in often rather complicated mixtures. While HPLC remains the most common technique for the analysis of benzodiazepines, ${ }^{7,9,14,15}$ mainly in reversed phase (RP) mode, several capillary zone electrophoresis (CZE) methods have recently been developed. ${ }^{2,16,17} \mathrm{CZE}$ represents an interesting alternative to HPLC for determination of pharmaceuticals, in general, ${ }^{18,19}$ mainly because of its higher efficiency in comparison with HPLC. ${ }^{20}$

The aim of this work was to develop and optimize separation conditions for a set of seven 1,4-BZDs in RP-
HPLC and CZE with UV detection and compare these methods in terms of efficiency, analysis time, resolution, elution order and basic validation parameters. The results aimed to answer the question if CZE is really a suitable alternative to RP-HPLC for the resolution/analysis of BZDs.

\section{EXPERIMENTAL}

\section{Chemicals}

Acetonitrile (ACN) for HPLC and methanol $(\mathrm{MeOH})$ for HPLC were obtained from Sigma-Aldrich (Steinheim, Germany). Glacial acetic acid (HAc, purity $>99.5$ $\%$ ), sodium dihydrogen phosphate anhydrous (purity $\geq$ $99 \%$ ) and mesityl oxide were purchased from Fluka (Buchs, Switzerland). Sodium hydroxide solution (0.1 $\mathrm{mol} / \mathrm{l}$ ) was from Agilent Technologies (Waldbronn, Germany). Ortho-phosphoric acid (85\%) was obtained from Lachema (Brno, Czech Republic). 1,4benzodiazepines, namely oxazepam, nitrazepam, lormetazepam, chlordiazepoxide, flunitrazepam, diazepam and bromazepam, were obtained from Sigma-Aldrich (Steinheim, Germany). The tablets of Diazepam Slova-

\footnotetext{
$\dagger$ Presented at the $10^{\text {th }}$ International Symposium and Summer School on Bioanalysis within the CEEPUS Network CII-HU-001004-0910, Zagreb, Croatia, July 2010.

* Author to whom correspondence should be addressed. (E-mail: kveta.kalikova@centrum.cz)
} 
kofarma ${ }^{\circledR} 5 \mathrm{mg}$ were product of Zentiva (Hlohovec, Slovakia) and Lexaurin ${ }^{\circledR} 3$ (bromazepamum $3 \mathrm{mg}$ ) was product of KRKA (Novo mesto, Slovenia). Water was prepared with a Milli-Q water purification system (Millipore, Milford, MA, USA). Stock solutions of the analyzed compounds were prepared in concentration of 1 $\mathrm{mg} / \mathrm{ml}$ using $\mathrm{MeOH}$ as a solvent.

\section{Equipment and Measurement Conditions}

All chromatographic measurements were performed on a Waters Breeze HPLC system (Waters Chromatography, Milford, MA, USA) consisting of a 1525 binary pump, a 717 plus autosampler, a column heater and a 2487 dual $\lambda$ absorbance detector. A Breeze software was used for process control and data handling. Columns used for separations were a Zorbax SB-C8 (column size $150 \mathrm{~mm} \times 4.6 \mathrm{~mm}$ i.d., particle size $5 \mu \mathrm{m}$ ) and Zorbax SB-C18 (column size $150 \mathrm{~mm} \times 4.6 \mathrm{~mm}$ i.d., particle size $5 \mu \mathrm{m}$ ), both purchased from Agilent Technologies (USA). The columns were thermostated at $25^{\circ} \mathrm{C}$. Flow rate was $2 \mathrm{ml} / \mathrm{min}$. Detection was carried out at the wavelength of $240 \mathrm{~nm}$. Mobile phases were composed of ACN/deionised water with acetic acid, $\mathrm{pH} 3.0$, in various ratios. Injected sample volume was $10 \mu \mathrm{L}$. Individual measurements were repeated three times.

Electrophoretic experiments were performed using an Agilent ${ }^{3 \mathrm{D}} \mathrm{CE}$ equipment operated under control of a ChemStation software (Agilent Technologies, Waldbronn, Germany). Detection was performed with a diode array detector at the detection wavelength of $240 \mathrm{~nm}$. A bare fused-silica capillary of $50 \mu \mathrm{m}$ i.d. and $363 \mu \mathrm{m}$ o.d. was product of Polymicro Technologies (Phoenix, USA). Total length of the capillary was $63.7 \mathrm{~cm}$ and length to the detector was $55.2 \mathrm{~cm}$. The capillary was thermostated at 25 ${ }^{\circ} \mathrm{C}$. Driving voltage was $+27 \mathrm{kV}$ (with cathode at the detector side). Samples were injected hydrodynamically: 50 mbar $\times 5 \mathrm{~s}$. Mesityl oxide was used as an electroosmotic flow (EOF) marker. Stock solutions of sodium dihydrogen phosphate and ortho-phosphoric acid at a concentration of $50 \mathrm{mM}$ were used for preparation of background electrolyte (BGE). The running buffer was prepared by mixing of appropriate amounts of the stock solutions and addition of water to obtain BGE with final composition of $6 \mathrm{mM}$ sodium dihydrogen phosphate and $33 \mathrm{mM}$ orthophosphoric acid. $\mathrm{pH}$ value of the BGE was 2.0. The capillary was rinsed with pure water for $10 \mathrm{~min}$, with $0.1 \mathrm{mM}$ $\mathrm{NaOH}$ for $10 \mathrm{~min}, 10 \mathrm{~min}$ with pure water again and 20 min with running buffer before use. Prior to each measurement the capillary was washed with the running buffer for $4.5 \mathrm{~min}$. All measurements were carried out in triplicates.

\section{Procedures}

Real Sample Preparation

Aliquots of powdered tablet samples were dissolved in methanol to concentration of $1 \mathrm{mg} / \mathrm{ml}$. The samples were sonicated for $20 \mathrm{~min}$ to provide complete dissolution. Appropriate volumes of clear supernatants were diluted with methanol to concentration of $0.01 \mathrm{mg} / \mathrm{ml}$ and with BGE to concentration of $0.05 \mathrm{mg} / \mathrm{ml}$ for HPLC and CZE analyses, respectively. The prepared samples were filtered through a $0.45 \mu \mathrm{m}$ filter before injection. The total amounts of the drugs were then determined using the optimized HPLC and CZE methods.

\section{Statistical Data Evaluation}

One-way analysis of variance (ANOVA) statistical method was used for robustness testing. Parameters, which could become significant sources of errors in practice, were chosen as variables. The significance level $(\alpha)$ was set at 0.05 . The one-way analysis of variance compares the medians of two or more groups in order to determine if at least a median value of one group is different from the others. The tests are nondirectional as the null hypothesis specifies that all medians are equal and the alternative hypothesis simply states that at least one median value is different. ${ }^{21}$ If the statistical $p$-value is higher than the chosen significance level, the null hypothesis of the equal medians is accepted.

\section{RESULTS AND DISCUSSION}

\section{Optimization of the Chromatographic Separation Conditions}

Optimization of separation conditions for seven drugs from 1,4-benzodiazepine group - bromazepam, oxazepam, nitrazepam, chlordiazepoxide, flunitrazepam, lormetazepam and diazepam - was carried out in RPHPLC system. ${ }^{2,7,14,15}$ The first column tested was Zorbax SB-C8 packed with an endcapped octyl-silica gel stationary phase. The most suitable mobile phase composition found in isocratic elution mode was ACN/deionised water with acetic acid, $\mathrm{pH} 3.0,30 / 70$ $(v / v)$. Baseline resolution of all seven analytes was achieved within 20 minutes - see Figure 1A. Compared to mobile phase composed just of $\mathrm{ACN}$ and deionised water the acidified mobile phase enhanced peak symmetry. Various linear gradient elution types were tested to decrease the analysis time. To preserve baseline resolution of a critical pair of flunitrazepam and lormetazepam the acetonitrile gradient could not be applied before $11^{\text {th }}$ minute. As the result of application of the gradient shorter analysis time was reached while the peak symmetry was preserved. However, the isocratic mode is more suitable for routine (repeated) measurements because it does not require column equilibration before each analysis.

Using column with more hydrophobic stationary phase octadecyl-silica gel SP - Zorbax SB-C18 - the analysis time was substantially reduced because a mo- 


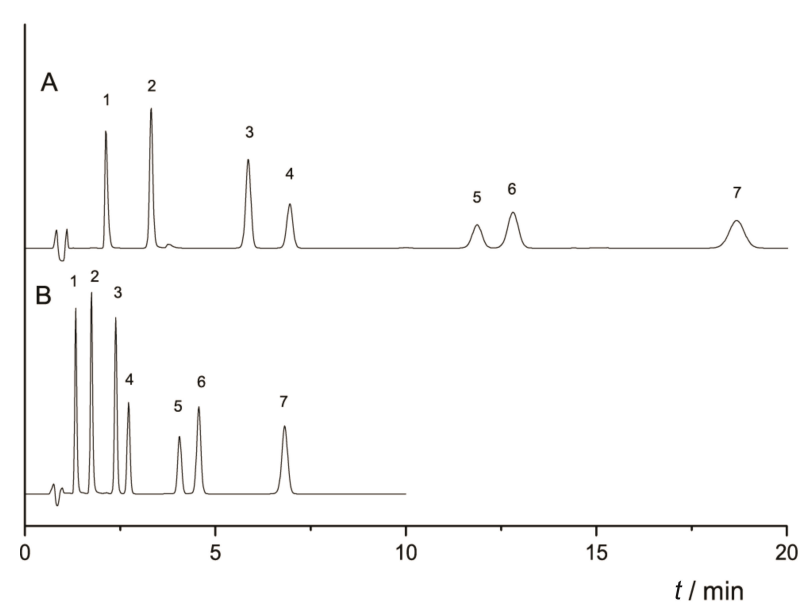

Figure 1. Chromatograms of 1,4-benzodiazepines separation. Chromatogram A: Zorbax SB-C8 column; mobile phase: ACN/water with HAc, pH 3.0, 30/70 $(v / v) ; 2 \mathrm{ml} / \mathrm{min} ; 25^{\circ} \mathrm{C}$; $240 \mathrm{~nm}$. Chromatogram B: Zorbax SB-C18 column; mobile phase: $\mathrm{ACN} /$ water with $\mathrm{HAc}, \mathrm{pH} 3.0,40 / 60(v / v) ; 2 \mathrm{ml} / \mathrm{min}$; $25{ }^{\circ} \mathrm{C} ; 240 \mathrm{~nm}$. Elution order: 1-chlordiazepoxide, 2bromazepam, 3-oxazepam, 4-nitrazepam, 5-flunitrazepam, 6lormetazepam, 7-diazepam.

bile phase with higher ACN content (40 volume percent) could be applied while the separation was still preserved. The baseline resolution of all seven 1,4BZDs was achieved within $7 \mathrm{~min}$ (Figure 1B). Linear gradient elution was also tested. Using the Zorbax SB$\mathrm{C} 18$ column and mobile phase composed of ACN/water with HAc, $\mathrm{pH} 3.0,40 / 60(v / v)$ and a linear gradient to volume fraction $\varphi=90 \%$ of acetonitrile applied from $2^{\text {nd }}$ to $3^{\text {rd }}$ minute, the analysis time was reduced to 4.5 minute. The results obtained under isocratic versus gradient elution conditions are summarized in Table 1 for comparison. The peak symmetry was similar under the both studied conditions. Therefore, the isocratic elution was selected as the optimized separation mode. The efficiency, expressed as plate number/meter of column, ranged under optimized isocratic conditions from $2.05 \times 10^{4}$ to $6.00 \times 10^{4}$ while under gradient elution conditions this parameter rendered higher values, from $1.97 \times 10^{4}$ to $3.55 \times 10^{5}$.

\section{Optimization of the Electrophoretic}

Separation Conditions

The software PeakMaster ${ }^{22}$ was utilized for selection of an initial BGE composition, $\mathrm{pH}$ and separation conditions. The optimized BGE composition for the analysis of BZDs was $6 \mathrm{mM}$ sodium and $39 \mathrm{mM}$ phosphate ions. According to PeakMaster calculations the optimized BGE had $\mathrm{pH} 2.0$, ionic strength $17.2 \mathrm{mM}$ and buffering capacity $48 \mathrm{mM}$. Nitrazepam was excluded from the studied set of analytes because of its instability in the buffer of the low $\mathrm{pH}$. Using $6 \mathrm{mM}$ phosphate buffer of higher $\mathrm{pH}$ the compounds were not baseline resolved. Baseline separation of six BZDs was obtained within 36 minutes with the BGE of $\mathrm{pH}$ 2.0. To reduce the analysis time additional pressure of 50 mbar was applied in $10^{\text {th }} \mathrm{min}$. Then the analysis time decreased to 17 minutes with baseline separation preserved - see Figure 2. The additional pressure could not be used earlier (before the $10^{\text {th }} \mathrm{min}$ ) because it would not allow baseline resolution of the first three compounds (bromazepam, diazepam and chlordiazepoxide). The efficiency, expressed as plate number / meter, ranged under optimized conditions from $1.11 \times 10^{5}$ to $3.08 \times 10^{5}$. The plate number of the lormetazepam peak was calculated to be an order of magnitude lower in comparison with all the other peaks obtained in the CZE measurements. This is caused by a resonance phenomenon ${ }^{23,24}$ that can lead to anomalous dispersion of the analyte's peak. ${ }^{25}$ The resonance can cause false results of analysis. It was described to have also a negative impact on efficiency of separation. ${ }^{26}$ Therefore, it is better to avoid using separation conditions at which the resonance occurs.

Table 1. Comparison of retention time $\left(t_{\mathrm{R}}\right)$, resolution $(R)$ and peak symmetry $(S)$ for isocratic and gradient elution with Zorbax SB C18 column. Mobile phase compositions: isocratic elution: ACN/deionised water with HAc, pH 3.0, 40/60 ( $v / v)$; gradient elution: ACN/deionised water with HAc, $\mathrm{pH} 3.0,40 / 60(v / v)$ in isocratic elution up to $2^{\text {nd }}$ minute and a linear gradient to volume fraction, $\varphi=90 \%$ of ACN applied from $2^{\text {nd }}$ to $3^{\text {rd }}$ mins

\begin{tabular}{clccccccc}
\hline & & \multicolumn{2}{c}{$t_{\mathrm{R}} / \mathrm{min}$} & \multicolumn{3}{c}{$R$} & & \\
\hline peak number & compound & iso & grad & iso & grad & iso & grad \\
\hline 1 & chlordiazepoxide & 1.32 & 1.31 & & & & 1.21 & 1.16 \\
2 & bromazepam & 1.76 & 1.76 & 4.44 & 4.33 & 1.12 & 1.14 \\
3 & oxazepam & 2.41 & 2.41 & 5.61 & 5.60 & 1.05 & 1.07 \\
4 & nitrazepam & 2.74 & 2.73 & 2.50 & 2.53 & 1.05 & 1.06 \\
5 & flunitrazepam & 4.09 & 3.90 & 8.39 & 10.03 & 1.03 & 0.99 \\
6 & lormetazepam & 4.60 & 4.06 & 2.65 & 1.75 & 1.01 & 1.04 \\
7 & diazepam & 6.83 & 4.40 & 9.07 & 4.36 & 1.02 & 1.01 \\
\hline
\end{tabular}




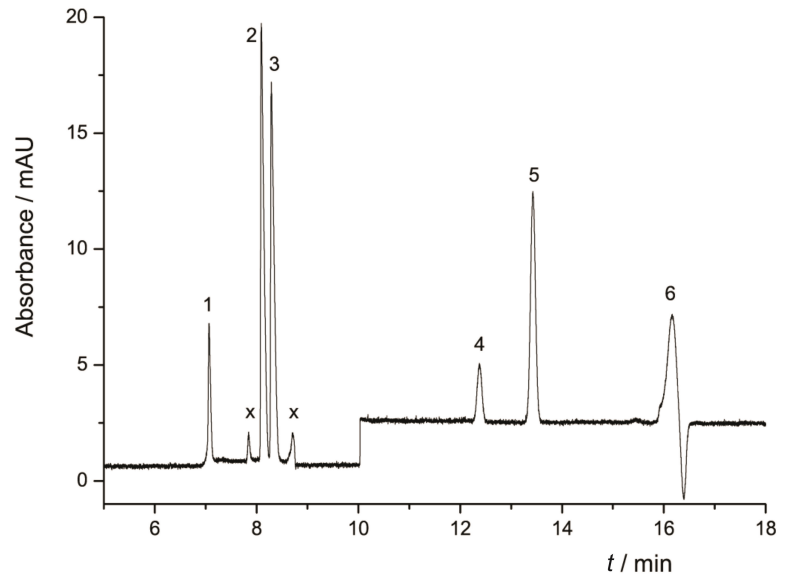

Figure 2. Electropherogram of 1,4-benzodiazepines separation. BGE: $6 \mathrm{mM}$ phosphate buffer, $\mathrm{pH} 2.0 ;+27 \mathrm{kV} ; 25^{\circ} \mathrm{C}$; $240 \mathrm{~nm}$. Elution order: 1-bromazepam, 2-diazepam, 3chlordiazepoxide, 4-flunitrazepam, 5-oxazepam, 6-lormetazepam in coincidence with stationary system peak, $\mathrm{x}$-impurities. Baseline "jump" at $10^{\text {th }} \mathrm{min}$ is caused by application of pressure.

\section{Validation of the Methods}

\section{Stability of Sample Solutions}

Stability of the sample solutions (1 mg/ml MeOH) kept at low temperature was tested by the optimized HPLC method during the period of three weeks. Almost all the samples were proved to be stabile over the three weeks period. The only exception was solution of chlordiazepoxide that showed slight instability after five days of storage.
However, it was still useful for the purpose of this study.

\section{Precision}

In order to evaluate the precision of the HPLC and CZE methods, repeatability and reproducibility of measurements were studied at the wavelength of $240 \mathrm{~nm}$. The repeatability of the retention factors and sample concentrations in HPLC and the effective mobilities and sample concentrations in CZE were determined as relative standard deviations (R.S.D.) for 10 consecutive injections of the set of 1,4-benzodiazepines at the concentrations of $0.01 \mathrm{mg} / \mathrm{ml}$ and $0.05 \mathrm{mg} / \mathrm{ml}$ in HPLC and $\mathrm{CZE}$, respectively. The reproducibility of the chromatographic and electrophoretic data (retention factor, effective mobility, sample concentration) of the tested set of analytes was measured in the period of 3 days by different scientists. The R.S.D. values for precision of the both methods are shown in Table 2. The results suggest that the HPLC and CZE methods are suitable for both qualitative and quantitative analysis of the 1,4-BZDs.

\section{Linearity}

The linearity was tested over the concentration range 0.001 to $0.1 \mathrm{mg} / \mathrm{ml}$ and 0.02 to $0.1 \mathrm{mg} / \mathrm{ml}$ in HPLC and $\mathrm{CZE}$, respectively. Measurements at all concentration levels were carried out in triplicate at six concentration levels and all values of peak areas were subjected to linear regression. Linear relationships between the peak areas in HPLC or the corrected peak areas (peak area/migration time) in CZE and the concentrations of individual 1,4-benzodiazepines were observed - see Table 3.

Table 2. Precision, expressed as relative standard deviation values of concentrations, retention factors and effective mobilities, of the developed HPLC and CZE methods for analysis of 1,4-BZDs

\begin{tabular}{|c|c|c|c|c|c|c|c|c|}
\hline & \multicolumn{4}{|c|}{ HPLC } & \multicolumn{4}{|c|}{ CZE } \\
\hline & \multicolumn{2}{|c|}{ Repeatability } & \multicolumn{2}{|c|}{ Reproducibility } & \multicolumn{2}{|c|}{ Repeatability } & \multicolumn{2}{|c|}{ Reproducibility } \\
\hline & R.S.D. $(c)$ & R.S.D. $(k)$ & R.S.D. $(c)$ & R.S.D. $(k)$ & R.S.D. $(c)$ & R.S.D. $\left(\mu_{\text {eff }}\right)$ & R.S.D. $(c)$ & R.S.D. $\left(\mu_{\text {eff }}\right)$ \\
\hline & $\%$ & $\%$ & $\%$ & $\%$ & $\%$ & $\%$ & $\%$ & $\%$ \\
\hline chlordiazepoxide & 0.965 & 0.229 & 1.66 & 0.635 & 3.94 & 0.950 & 14.7 & 2.06 \\
\hline bromazepam & 0.999 & 0.188 & 1.81 & 0.262 & 4.11 & 0.743 & 9.09 & 1.11 \\
\hline oxazepam & 0.985 & 0.108 & 1.37 & 0.245 & 4.74 & 0.732 & 8.12 & 0.326 \\
\hline nitrazepam & 0.943 & 0.145 & 1.18 & 0.313 & $\mathrm{x}$ & $\mathrm{x}$ & $\mathrm{x}$ & $\mathrm{x}$ \\
\hline flunitrazepam & 1.24 & 0.131 & 1.30 & 0.261 & 4.11 & 0.486 & 8.87 & 0.439 \\
\hline lormetazepam & 1.14 & 0.123 & 1.70 & 0.227 & $\mathrm{x}$ & $\mathrm{x}$ & $\mathrm{x}$ & $\mathrm{x}$ \\
\hline diazepam & 1.07 & 0.132 & 2.20 & 0.362 & 4.10 & 0.903 & 11.2 & 1.19 \\
\hline
\end{tabular}

$c$, concentration; $k$, retention factor; $\mu_{\text {eff }}$, effective mobility; R.S.D, relative standard deviation.

repeatability - the R.S.D. values were calculated from ten consecutive injections of the mixture of analytes.

reproducibility - the R.S.D. values were calculated from measurements obtained by different scientists within a period of 3 days. 
Table 3. Linearity - parameters of the obtained linear regression equations

\begin{tabular}{|c|c|c|c|c|c|c|c|c|}
\hline & \multicolumn{4}{|c|}{ HPLC } & \multicolumn{4}{|c|}{ CZE } \\
\hline & $\frac{\text { slope }}{10^{3} \mathrm{mV} \mathrm{s} \mathrm{ml} \mathrm{mg}^{-1}}$ & $\frac{\text { intercept }}{\mathrm{mVs}}$ & $R$ & S.D. & $\frac{\text { slope }}{\mathrm{mAU} \mathrm{ml} \mathrm{mg}^{-1}}$ & $\frac{\text { intercept }}{10^{-3} \mathrm{mAU}}$ & $R$ & $\frac{\text { S.D. }}{10^{-3}}$ \\
\hline chlordiazepoxide & 2.85 & -56 & 0.9992 & 41.5 & 2.85 & 5 & 0.9919 & 14.5 \\
\hline bromazepam & 2.80 & -7.2 & 0.9999 & 4.41 & 1.09 & 5 & 0.9949 & 3.79 \\
\hline oxazepam & 23.32 & -4.7 & 0.9999 & 3.37 & 1.19 & 1 & 0.9987 & 2.62 \\
\hline nitrazepam & 15.93 & 4 & 0.9999 & 7.54 & $\mathrm{x}$ & $\mathrm{x}$ & $\mathrm{x}$ & $\mathrm{x}$ \\
\hline flunitrazepam & 13.49 & -3.6 & 0.9999 & 3.04 & 0.41 & 0.4 & 0.9956 & 1.38 \\
\hline lormetazepam & 21.77 & -10 & 0.9999 & 12.0 & $\mathrm{x}$ & $\mathrm{x}$ & $\mathrm{x}$ & $\mathrm{x}$ \\
\hline diazepam & 26.9 & 5 & 0.9998 & 11.8 & 2.34 & 3 & 0.9982 & 5.40 \\
\hline
\end{tabular}

Table 4. LOD and LOQ data under optimized separation conditions - see the text

\begin{tabular}{lcccc}
\hline & \multicolumn{2}{c}{ HPLC } & \multicolumn{2}{c}{ CZE } \\
\hline \multicolumn{1}{c}{ LOD $/ \mathrm{ng} \mathrm{ml}^{-1}$} & LOQ $/ \mathrm{ng} \mathrm{ml}^{-1}$ & $\mathrm{LOD} / \mu \mathrm{g} \mathrm{ml}{ }^{-1}$ & $\mathrm{LOQ} / \mu \mathrm{g} \mathrm{ml}{ }^{-1}$ \\
chlordiazepoxide & 8.68 & 28.9 & 1.63 & 5.44 \\
bromazepam & 7.89 & 26.3 & 4.27 & 14.3 \\
oxazepam & 8.91 & 29.7 & 2.98 & 9.94 \\
nitrazepam & 17.1 & 56.8 & $\mathrm{x}$ & $\mathrm{x}$ \\
flunitrazepam & 26.7 & 89.0 & 11.2 & 37.3 \\
lormetazepam & 17.8 & 59.2 & $\mathrm{x}$ & $\mathrm{x}$ \\
diazepam & 22.8 & 75.9 & 1.57 & 5.22 \\
\hline
\end{tabular}

$\mathrm{x}$ - not quantified.

\section{Limit of Detection and Limit of Quantification}

The limit of detection (LOD), expressed as a concentration at a signal-to-noise ratio 3:1, was calculated on the basis of the baseline noise, which was evaluated by recording the detector response over a period of approximately ten times the widths of the peaks. The signal-to-noise ratio of 10:1 was used to determine the limit of quantification (LOQ). LODs and LOQs were calculated for all seven 1,4-benzodiazepines under the optimized isocratic HPLC conditions. Under the optimized separation conditions in CZE LODs and LOQs were determined for the five separated BZDs. It was not possible to quantify the peak of lormetazepam because its mobility (at the $\mathrm{pH}$ of the BGE) was very close to zero and therefore close to the mobility of the stationary system peak. The observed resonance substantially changed the peak height (area), which could not be used anymore for quantification of the analyte. The LODs and LOQs data are summarized in Table 4.

\section{Robustness Studies}

One-way ANOVA statistical method was used for robustness testing. The selected variable parameters of the HPLC method were: column temperature $\left(24^{\circ} \mathrm{C}, 25^{\circ} \mathrm{C}\right.$ and $\left.26^{\circ} \mathrm{C}\right), \mathrm{pH}$ of the aqueous part of the mobile phase $(2.8,3.0$ and 3.2) and acetonitrile content in the mobile phase $(40 \% \pm 2 \%)$. The tested parameters of the CZE method were: temperature $\left(24{ }^{\circ} \mathrm{C}, 25^{\circ} \mathrm{C}\right.$ and $\left.26^{\circ} \mathrm{C}\right)$ and background electrolyte $\mathrm{pH}(2.0 \pm 0.2 \mathrm{pH}$ units $)$. The robustness was determined from triplicate injections of $0.01 \mathrm{mg} / \mathrm{ml}$ of the set of seven 1,4-BZDs and of 0.05 $\mathrm{mg} / \mathrm{ml}$ of the set of five 1,4-BZDs in HPLC and CZE, respectively, for every change of the tested parameters. The effect of the method parameters on retention factors or effective mobilities and analytes concentrations was calculated. The hypothesis that errors resulted from a normal distribution was tested first. This hypothesis was accepted in all cases (at $\alpha=0.05$ ). Consequently, the robustness of the method was examined using the oneway ANOVA - see Experimental. The null hypothesis 


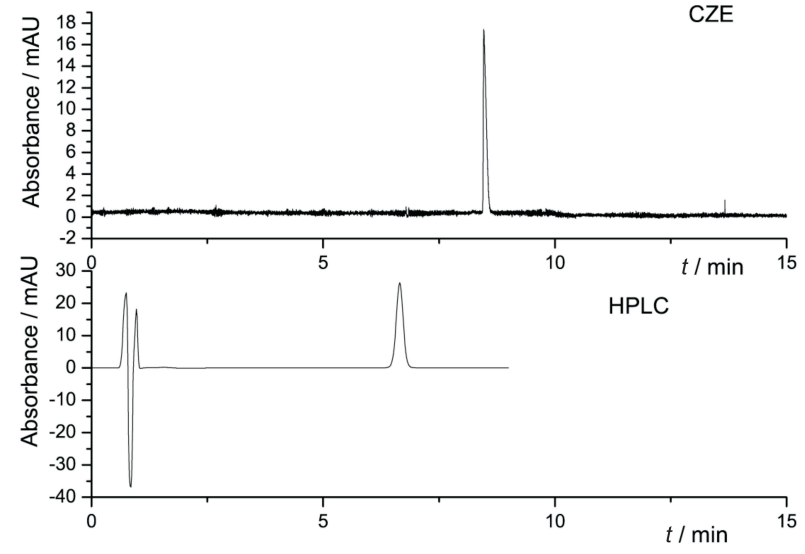

Figure 3. Reports of diazepam tablets analyses under optimized CZE and HPLC conditions. For details see captions to Figures 1 and 2 or Experimental.

was accepted in almost all cases (obtained $p$-values were higher than 0.05 ), so the robustness of the selected parameters of the methods was verified. An exception was the background electrolyte $\mathrm{pH}$. The effect of an increase of the BGE $\mathrm{pH}$ value on the effective mobility and concentration of chlordiazepoxide and diazepam could not be evaluated by one-way ANOVA due to their co-migration.

\section{Real Sample Analysis - Accuracy}

Two different tablets containing $10 \mathrm{mg}$ of diazepam and $3 \mathrm{mg}$ of bromazepam were analyzed three times in concentrations of $0.01 \mathrm{mg} / \mathrm{ml}$ and $0.05 \mathrm{mg} / \mathrm{ml}$ using the optimized HPLC and CZE methods, respectively. Accuracy of the methods, regarded as the closeness of agreement between the claimed contents of tablets and the found values, were $103.2 \%$ for bromazepam and $102.9 \%$ for diazepam by the HPLC method. Accuracy of the CZE analyses were $109.8 \%$ for bromazepam and $103.2 \%$ for diazepam. The reports of the analyses of diazepam are shown in Figure 3. The same results can be achieved for other pharmaceutical preparations containing BZDs.

\section{Comparison of HPLC and CZE Methods}

RP-HPLC and CZE methods for separation of 1,4benzodiazepines were developed and optimized. The HPLC analysis under isocratic elution conditions can be achieved within 7 mins, application of mobile phase gradient can reduce the analysis time to 4.5 minutes. Separation of 1,4-benzodiazepines requires separation time of 17 minutes in the optimized CZE system if additional pressure is applied. The LOD and LOQ values are approximately three orders of magnitude lower in HPLC than in CZE. There are two reasons for these quantification results in $\mathrm{CZE}$ : (i) the baseline noise is much higher in CZE than in HPLC, and (ii) lower sample volume is injected in CZE $(6 \mathrm{nl})$ in comparison with
HPLC $(10 \mu \mathrm{l})$. On the other hand the plate number / meter is an order of magnitude higher in CZE than in HPLC. Narrower profiles of peaks obtained in capillary zone electrophoresis can make easier and more reliable quantification from the peak height (compare the analyte's peaks in Figure 3).

\section{CONCLUSION}

HPLC and CZE were proved to be suitable methods for separation of benzodiazepines and for their qualitative and quantitative analyses in pharmaceutical formulations. From the results obtained it is obvious that the method of first choice is RP-HPLC.

Acknowledgements. Financial supports of the Grant Agency of the Czech Republic, grant No. 203/08/1428, CEEPUS, grant No. CII-HU-0010-04-0910, the Grant Agency of the Charles University, grant No. 51009 and the long-term research plan of the Ministry of Education of the Czech Republic, No. MSM 0021620857 are gratefully acknowledged. The authors want to express their gratitute Renata Gilar for language corrections.

\section{REFERENCES}

1. L. A. Berrueta, B. Gallo, and F. Vicente, J. Pharm. Biomed. Anal. 10 (1992) 109-136.

2. S. McClean, E. O'Kane, J. Hillis, and W. F. Smyth, J. Chromatogr. A 838 (1999) 273-291.

3. A. EL Mahjoub and C. Staub, J. Chromatogr. B 742 (2000) 381-390.

4. E. Lavie, M. Fatséas, C. Denis, and M. Auriacombe, Drug Alcohol Depend. 99 (2009) 338-344.

5. Z. Es'haghi, L. Daneshvar, P. Salari, and S. Bandegi, Chemija 20 (2009) 180-185.

6. T. Ishida, Y. Obara, and C. Kamei, J. Pharmacol. Sci. 111 (2009) 44-52.

7. L. Mercolini, R. Mandrioli, M. Amore, and M. A. Raggi, J. Sep. Sci. 31 (2008) 2619-2626.

8. S. J. Marin, R. C. M. Merrell, and G. A. McMillin, J. Anal. Toxicol. 32 (2008) 491-498.

9. V. F. Samanidou, A. P. Pechlivanidou, and I. N. Papadoyannis, J. Sep. Sci. 30 (2007) 679-687.

10. O. F. Drummer, J. Chromatogr. B 713 (1998) 201-225.

11. A. A. Salem, B. N. Barsoum, and E. L. Izake, Anal. Chem. Acta 498 (2003) 79-81.

12. K. H. Beyer and S. Martz, Arch. Pharm. 324 (1991) 933-935.

13. A. Elbrashy, F. A. Aly, and F. Belal, Microchim. Acta 110 (1993) 55-60.

14. O. Quintela, F.-L. Sauvage, F. Charvier, J.-M. Gaulier, G. Lachatre, and P. Marquet, Clin. Chem. 52 (2006) 1346-1355.

15. P. R. Puopolo, M. E. Pothier, S. A. Volpicelli, and J. A. Flood, Clin. Chem. 37 (1991) 701-706.

16. G. McGrath, S. McClean, E. O'Kane, W. F. Smyth, and F. Tagliaro, J. Chromatogr. A 735 (1996) 237-241.

17. R. Webb, P. Doble, and M. Dawson, Electrophoresis 28 (2007) 3553-3565.

18. T. K. Natishan, J. Liq. Chromatogr. RT 28 (2005) 1115-1160.

19. A. Jouyban and E. Kenndler, Electrophoresis 29 (2008) 3531-3551.

20. M. C. V. Mamani, J. A. Farfán, F. G. R. Reyes, and S. Rath, Talanta 70 (2006) 236-243. 
21. J. L. Hintze, NCSS User's Guide II, NCSS, Kaysville, UT, USA, 2007.

22. B. Gaš, M. Jaroš, V. Hruška, I. Zusková, and M. Štědrý, $L C G C$ Europe 18 (2005) 282-288.

23. M. Šť̌drý, M. Jaroš, and B. Gaš, J. Chromatogr. A 960 (2002) 187-198.
24. B. Gaš, V. Hruška, M. Dittmann, F. Bek, and K. Witt, J. Sep. Sci. 30 (2007) 1435-1445.

25. J. L. Beckers, P. Gebauer, and P. Boček, J. Chromatogr. A 916 (2001) 41-49.

26. B. Gaš and E. Kenndler, Electrophoresis 25 (2004) 3901-3912. 